\title{
EL CAMINO HACIA LA SOSTENIBILIDAD EN EL PAÍS VASCO. LA AGENDA 21 EN VITORIA-GASTEIZ ${ }^{* 1}$
}

\author{
Galdos Urrutia, R. \& Ruiz Urrestarazu, E. \\ Dpto. de Geografía, Prehistoria y Arqueología. \\ Universidad del País Vasco. C/Tomás y Valiente, s/n. 01006 Vitoria-Gasteiz \\ E-mail: fgpgaurr@vc.ehu.es
}

\begin{abstract}
Resumen: El artículo aborda las actuaciones llevadas a cabo por el Departamento de Ordenación del Territorio y Medio Ambiente del Gobierno Vasco para impulsar la implantación de la Agenda 21 Local en los municipios vascos. Se hace especial hincapié en la promoción del trabajo en red a través de la cooperación intermunicipal. Como estudio de caso, se ha elegido el municipio de Vitoria-Gasteiz, pionero en la región en la puesta en marcha de dicha Agenda 21 Local, lo que permite realizar una primera valoración de sus logros y fracasos.
\end{abstract}

Palabras clave: Agenda 21 Local, Indicadores de Sostenibilidad, País Vasco, Vitoria-Gasteiz.

Summary: The article deals with actions carried out by the Department of Environment and Regional Planning for the Basque Government in order to support the introduction of Local Agenda 21 in Basque municipalities. Special emphasis is placed on networking via intermunicipal cooperation. By way of a case study, the municipal area of Vitoria-Gasteiz -pioneer in the region for implementing the said Local Agenda 21- has been chosen, providing an opportunity to make an initial assessment of its achievements and failures.

Keywords: Local Agenda 21, Sustainability Indicators, Basque Country, VitoriaGasteiz.

* Recibido: 1-9-05. Aceptado: 22-12-05.

1 Este artículo ha sido realizado en el marco de un proyecto de Investigación sobre Estrategias de cooperación y desarrollo sostenible en el País Vasco y Navarra, financiado por el Ministerio de Ciencia y Tecnología (Referencia: BSO2002-04233-C10-04). 


\section{Origen y desarrollo de la Agenda 21 Local}

En 1989 la Asamblea General de las Naciones Unidas acordó la celebración de una Conferencia sobre Medio Ambiente y Desarrollo, la cual tuvo lugar en 1992 en la ciudad brasileña de Río de Janeiro (Cumbre de Río o Cumbre de la Tierra). Participaron 175 delegaciones nacionales, convirtiéndose en la primera reunión internacional de dicha magnitud realizada después del final de la guerra fría; en ella se comprometieron a promover el desarrollo sostenible. Entre sus logros figuran la concertación de dos acuerdos internacionales (Convenio Marco sobre el Cambio Climático y Convenio sobre la Biodiversidad), la formulación de una Declaración de principios y la elaboración de un Programa de acción sobre Desarrollo Mundial Sostenible, más conocido como Programa o Agenda 21.

El Programa 21 es fundamentalmente una guía para la aplicación de un nuevo modelo de desarrollo, un modelo que se define por su sostenibilidad en el manejo de los recursos naturales, en la preservación de la biodiversidad, ecuánime y justo tanto en las relaciones económicas entre las naciones como en la distribución de la riqueza nacional entre los diferentes segmentos sociales, económicamente eficiente y políticamente participativo y democrático. En el Preámbulo, se exhorta a los gobiernos a que adopten estrategias nacionales para el desarrollo sostenible que deben elaborarse con una amplia participación de todos los sectores; se reconoce el papel de vanguardia de los gobiernos nacionales en el proceso de cambio pero se insiste en la necesidad de que actúen en asociación con organizaciones internacionales, con autoridades regionales y locales así como con asociaciones civiles. Al alentar la participación más amplia del público y la participación activa de las organizaciones no gubernamentales, el Programa 21 apuesta de forma decidida por reforzar la sociedad civil, la responsabilidad cívica y por que los ciudadanos dejen de ser sujetos pasivos y se incorporen al esfuerzo de la sostenibilidad (Riego Artigas, 2004). El Programa 21 es un plan de objetivos amplios que propone una serie de políticas a llevar a cabo en todas aquellas áreas relacionadas con el desarrollo sostenible. Se estructura en 4 secciones: 1) Dimensiones sociales y económicas, 2) Conservación y gestión de los recursos, 3) Fortalecimiento del papel de los grupos sociales, y 4) Medios para la puesta en práctica.

Aunque es un plan de acción mundial para el desarrollo sostenible, debido a las interrelaciones existentes entre los procesos globales y las acciones locales, los logros a escala global requieren logros a escala local. Es por este motivo que el Programa 21 atiende la escala local a la que dedica de forma exclusiva el capítulo 28 Autoridades locales y Agenda 21, que se incluye en la sección 3. En su punto 1 se señala que "Como tantos de los problemas y de las soluciones de que se ocupa la Agenda 21 se relacionan con las actividades locales, la participación y cooperación de las autoridades locales constituirán un factor determinante para el logro de los 
objetivos del Programa" y añade que las autoridades locales, al ser las más cercanas a los ciudadanos, "desempeñan una función importantísima en la educación y movilización del público en pro del desarrollo sostenible". Este capítulo contiene además una propuesta de objetivos y de actividades a desarrollar. Así, se propone que para el año 1996 "la mayoría de las autoridades locales de cada país deberían haber llevado a cabo un proceso de consultas con sus respectivas poblaciones y haber logrado un consenso sobre una Agenda 21 Local para la comunidad", objetivo que ha estado lejos de alcanzarse; igualmente se propone incrementar la cooperación y coordinación entre autoridades locales con el fin de aumentar el intercambio de información y experiencias. Del mismo modo se insta a las autoridades locales para que ejecuten y supervisen programas encaminados a lograr que las mujeres y los jóvenes estén representados en los procesos de adopción de decisiones, planificación y ejecución. Como actividades a desarrollar se propone que cada autoridad local inicie un diálogo con sus ciudadanos, organizaciones locales y empresas privadas y apruebe una Agenda 21 Local (A21L).

En Europa el proceso de implantación de la A21L arranca con la $1^{a}$ Conferencia de Ciudades y Pueblos Europeos Sostenibles celebrada en 1994 en la ciudad danesa de Aalborg, impulsada por el International Council for Local Environmental Initiatives (ICLEI), organismo creado en 1990 con el objetivo de aglutinar a nivel internacional a todos los gobiernos locales, regionales y nacionales comprometidos con el desarollo sostenible. Este organismo ha tenido un papel destacado en la implantación de la A21 L. A Aalborg acudieron 80 autoridades locales y 253 representantes de organizaciones internacionales y miembros de administraciones y entidades privadas, los cuales aprobaron la Carta de las ciudades europeas bacia la sostenibilidad o Carta de Aalborg. Este documento consta de tres partes. En la primera, Declaración de consenso. Las ciudades europeas hacia la sostenibilidad, las ciudades firmantes se comprometen a integrar los principios de sostenibilidad en todas las políticas locales con el fin de conseguir una equidad social, una economía sostenible y la sostenibilidad ambiental y a seguir el mandato del Programa 21 de trabajar con todos los sectores (ciudadanos, empresas, grupos de interés) en el desarrollo de las A21Ls. En la segunda parte, Campaña de las ciudades europeas sostenibles, se responsabilizan a "trabajar juntas para un desarrollo sostenible en un proceso de aprendizaje a partir de la experiencia y de los éxitos conseguidos a escala local" y a poner en marcha la campaña de ciudades europeas sostenibles para animar y apoyarlas en su tarea a favor de la sostenibilidad. La tercera parte, Implicación en el proceso de las Agenda 21 Locales: Planes de acción local a favor de la sostenibilidad, recoge el compromiso de las ciudades de alcanzar un consenso sobre la A21L antes de finalizar el año 1996, de acuerdo con el mandato establecido en el capítulo 28 del Programa 21. La Carta de Aalborg fue firmada inicialmente por 80 municipios europeos.

Dos años más tarde, en 1996, se celebró en Lisboa la $2^{a}$ Conferencia Europea sobre Ciudades y Pueblos Sostenibles en la que participaron 1.000 representantes de 
autoridades locales y regionales de toda Europa, que conocieron la situación del proceso de A21L en 35 países europeos y refrendaron el documento Plan de Acción de Lisboa: de la Carta a la Acción, también conocido como Carta de Lisboa. Este documento confirma que la Carta de Aalborg es uno de los mejores puntos de inicio para un proceso de A21L y que deben ser las autoridades locales las principales dinamizadoras del proceso. También se propone el establecimiento de alianzas entre autoridades, en forma de asociaciones, redes y campañas con el fin de ganar fortaleza. Para esa fecha la Carta de Aalborg había sido firmada por 250 autoridades locales y regionales.

En la $3^{a}$ Conferencia, en Hannover en el año 2000, se realizó un balance sobre la Campaña de las ciudades europeas sostenibles y se aprobó la Declaración de Hannover de los Lideres europeos municipales en el umbral del siglo XXI. Con este nuevo documento los participantes declaran su apoyo a la integración europea y a la ampliación de la UE; exhortan a las instituciones europeas a dar prioridad al desarrollo local sostenible y a los gobiernos nacionales a apoyar la implantación de la Agenda 21 y acuerdan el establecimiento de un conjunto de indicadores europeos comunes que permitan comparar los cambios realizados entre las ciudades europeas.

Al cabo de diez años de la primera reunión, en 2004, se eligió de nuevo la ciudad de Aalborg para celebrar la $4^{a}$ Conferencia de las ciudades europeas sostenibles, conocida como Aalborg +10 , en la que se realizó una revisión de los 10 años de trabajo dedicados a la sostenibilidad local, evaluándose los avances y dificultades del proceso. En esta ocasión los cerca de 1.000 representantes que acudieron firmaron un documento conocido como los Compromisos de Aalborg que consta de 10 puntos los cuales hacen referencia a una serie de aspectos en los que se quiere avanzar: participación ciudadana, gestión urbana, gestión eficiente de recursos naturales, consumo responsable, planificación urbana que evite la urbanización difusa y promoción de la arquitectura sostenible, mejor movilidad y menos tránsito, acción local para la salud, economía sostenible, equidad social y responsabilidad local ante el cambio climático. Con anterioridad se había adoptado en 2001 a propuesta de la Comisión Europea la Estrategia Europea para un desarrollo sostenible. Supone una apuesta por una actuación política a largo plazo que sea capaz de hacer frente a las consideradas principales amenazas al desarrollo sostenible (calentamiento global, pobreza y exclusión social, envejecimiento de la población, pérdida de biodiversidad, congestión en el transporte y desequilibrios regionales). La estrategia incluye por un lado una serie de propuestas y recomendaciones transversales encaminadas a mejorar la eficacia de la actuación política y a crear las condiciones para el desarrollo sostenible, y por otro una serie de objetivos primordiales y medidas específicas a escala comunitaria para responder a los principales retos del desarrollo sostenible en Europa. Recogen los compromisos adquiridos en la cumbre de Lisboa de 2000 en el ámbito de la política social (medidas contra la pobreza y la exclusión social y medidas para hacer frente a las repercusiones económicas y sociales derivadas del proceso de envejecimiento) a 
los que añade nuevos objetivos dirigidos a limitar el cambio climático e incrementar el uso de energías limpias, a actuar en una amplia gama de políticas con un enfoque global y transectorial, a dar respuesta a las amenazas a la salud pública, a gestionar de modo más responsable los recursos naturales y a mejorar el sistema de transporte y la ordenación territorial.

\section{La Agenda 21 Local en el País Vasco}

El concepto de sostenibilidad aparece por primera vez en la legislación vasca en el año 1998, cuando se aprueba la Ley General 3/1998 de Protección del Medio Ambiente del País Vasco, en la que se expresa la decisión de adoptar un modelo de desarrollo sostenible (Echebarria \& Aguado, 2004). Tres años más tarde en el Compromiso por la Sostenibilidad del País Vasco del año 2001 se expresa de nuevo el acuerdo firme para avanzar hacia un nuevo modelo de desarrollo basado en la sostenibilidad, mediante la aplicación de principios que han de definir la estrategia acordada para el País Vasco, tales como la solidaridad intergeneracional e internacional, la cohesión y participación social, el principio de precaución, la necesidad de integrar la variable ambiental en todas las políticas sectoriales y la apuesta por la ecoeficiencia. En el año 2002 se da un paso más al aprobarse la Estrategia ambiental vasca de desarrollo sostenible 2002-2020, que establece una serie de metas ambientales a largo plazo que debe alcanzar la sociedad para garantizar un nivel óptimo de calidad de vida para las generaciones actuales y las futuras. La elección del año 2020 como horizonte estratégico hace coincidir la Estrategia vasca con la Estrategia Europea para un Desarrollo Sostenible. Las metas propuestas son cinco: garantizar un agua, aire y suelos limpios y saludables; una gestión responsable de recursos naturales y residuos; protección de la naturaleza y biodiversidad; equilibrio territorial y movilidad y limitar la influencia del cambio climático. Se establecen una serie de acciones y un total de 223 compromisos para cuya realización se fija un horizonte temporal. Uno de estos compromisos obliga a que todos los municipios vascos de más de 5.000 habitantes deberían tener diseñado su programa de A21L para el año 2006, ya sea de manera individualizada o comarcal.

La promoción de la A21L ha sido asumida por el Departamento de Ordenación del Territorio y Medio Ambiente conjuntamente con IHOBE (Sociedad Pública de Gestión Ambiental) (Echebarria \& Aguado, 2002). Los resultados han superado con creces las previsiones. A mediados de 2005 un total de 192 municipios están trabajando en fases más o menos avanzadas en la implantación de la $\mathrm{A} 21 \mathrm{~L}$, lo que supone cerca del $78 \%$ de todos los municipios vascos. Esta cifra afecta por tanto no sólo a aquellos municipios que cuentan con más de 5.000 habitantes, 64 en total, sino que también incluye a otros 130 de tamaño demográfico inferior. El avance que se ha 
dado en pocos años es notable: en el año 2002 sólo 18 municipios estaban implicados en la A21L; en 2005, 192.

Dado el escaso tamaño de la mayoría de los municipios vascos, sus ayuntamientos no disponen de los recursos humanos, técnicos y económicos necesarios para poner en marcha un proceso de A21L. Muchos de ellos, por ejemplo, carecen de técnicos municipales en medio ambiente. Para superar estas carencias estructurales se ha propiciado el trabajo en grupo, en asociación y red, siguiendo las propuestas emitidas por la Segunda Conferencia de Lisboa. En este sentido, las convocatorias anuales del Departamento de Ordenación del Territorio y Medio Ambiente que se pusieron en marcha en 1998 para acceder a subvenciones para el diseño, desarrollo y/o ejecución de Planes Locales de Acción Ambiental, han ido dirigidas tanto a ayuntamientos como a asociaciones municipales (caso de Mancomunidades). Asimismo, en la Guía práctica para la implantación y desarrollo de la Agenda Local 21 en los municipios de Euskadi elaborada por el mismo Departamento en el año 2000 se insiste en el papel relevante que en el proceso de implantación y desarrollo de la A21L pueden tener las agrupaciones municipales (Mancomunidades y Cuadrillas) como entidades locales territoriales con competencias y servicios delegados por los municipios. En concreto se les asignan dos funciones: impulsar y colaborar en la implantación de la A21L. Se reconoce que la labor de estas agrupaciones beneficia a todos los municipios que las integran pero sobre todo a los más pequeños.

La promoción del trabajo en grupo se pone en marcha por parte del Gobierno Vasco con el Programa de Promoción de Agenda 21 Locales en Municipios Vascos 2000-2003 dentro del cual se configura el organismo Udaltalde 21. Este se define como un grupo de trabajo formado por diferentes municipios (entre 4 y 15) con el objetivo común de implantar la A21L. Un Udaltalde está integrado por:

- una serie de municipios que se comprometen a iniciar el proceso de A21L

- una entidad coordinadora, que puede ser una agencia de desarrollo comarcal o una entidad local territorial (mancomunidad o cuadrilla) y que será la encargada de dinamizar y coordinar las actividades, gestionar el presupuesto para la puesta en marcha de las actuaciones comunes y apoyar a los municipios de menor tamaño

- la Sociedad Pública IHOBE y la Diputación correspondiente, que serán los organismos encargados de promover la constitución de Udaltaldes, de fomentar la colaboración y participación entre el grupo de municipios y de trasmitir las experiencias de otros Udaltaldes.

De forma experimental en el año 2000 se puso en marcha el primer Udaltalde 21, un proyecto piloto en el que participaron 9 municipios de las tres provincias: dos de Álava (Amurrio y Llodio), tres de Vizcaya (Basauri, Erandio y Santurtzi) y cuatro de 
Guipúzcoa (Azkoitia, Azpeitia, Legazpi y Zarautz). Este Udaltalde estuvo en funcionamiento hasta el año 2001 momento en que cada uno de los municipios citados elaboraron su Plan de Acción Local (PAL). Finalizada esta experiencia piloto, las administraciones a partir de 2002 han promovido la creación de nuevos Udaltaldes en los que se han ido integrando la mayoría de los municipios vascos. Hasta mediados de 2005 se habían creado 21 Udaltaldes, en los que participan o han participado un total de 184 municipios (mapa 1). En la actualidad sólo funcionan catorce pues los siete restantes se han disuelto una vez que han cumplimentado las fases iniciales del proceso de A21L: diagnóstico ambiental, social y económico, formulación y priorización de objetivos, elaboración del PAL y puesta en marcha de canales estables de participación ciudadana. Las entidades colaboradoras integradas en los Udaltaldes son de naturaleza diversa: en alguno este papel le corresponde a Mendikoi (Sociedad pública del Gobierno Vasco para la Formación, Promoción y Desarrollo Rural); en otros casos son Mancomunidades o Cuadrillas; lo más frecuente es que sean agencias de desarrollo las que asuman las funciones de entidad colaboradora. El número de municipios implicados ayuda a dimensionar la importancia del proceso. De un total de 192 municipios que están desarrollando en diferente fases la A21L, 182 están o han estado agrupados en Udaltaldes, 10 trabajan de forma individual (en Bizkaia son los de Bilbao, Amorebieta-Etxano, Barakaldo, Getxo y Leioa; en Gipuzkoa, San Sebastián y Tolosa; y en Álava, Vitoria-Gasteiz, Valdegovía y Lantarón) y 57 no han iniciado todavía este proceso.

La método de trabajo es común a todos los Udaltaldes durante su periodo de vigencia que no debe superar los 18 meses. En ese año y medio de vida, cada Udaltalde debe completar una serie de fases que culminan con la implantación y el seguimiento de la A21L:

a) Fase pre-operacional: con una duración de tres meses, es la fase en la que, una vez que los municipios han adoptado un compromiso serio con la Sostenibilidad, se firma el convenio para la creación del Udaltalde.

b) Fase de diseño: en los 15 meses siguientes cada municipio del Udaltalde debe poner en marcha mecanismos para la participación e implicación de los técnicos municipales de los distintos departamentos, elaborar un diagnóstico sobre la situación ambiental del municipio, formular líneas estratégicas para la resolución de los problemas ambientales detectados y establecer una serie de indicadores de sostenibilidad. Por último se deben concretar los objetivos en una serie de actuaciones que conformarán el PAL. Es en esta fase, en concreto en la subfase de diagnóstico ambiental, económico y social cuando se incorpora la participación ciudadana.

c) Fase de implantación y seguimiento: a partir del año y medio, con la implantación del PAL se abre una nueva etapa que requiere su seguimiento y control mediante los indicadores de sostenibilidad que permitirán evaluar el grado de eficacia de las 
acciones implementadas. Es en este momento cuando cesa la labor del Udaltalde y por tanto se procede a su disolución.

El PAL, definido como un instrumento para orientar la gestión y las políticas locales en pro de la sostenibilidad, se constituye en el elemento fundamental de la A21L (IHOBE, 2004). A diferencia de otros instrumentos de planificación utilizados por los municipios, el PAL se caracteriza por integrar el desarrollo sostenible como objetivo fundamental y la participación ciudadana como elemento intrínseco del proceso de planificación. En su elaboración el PAL debe potenciar su carácter sostenible, estratégico, transversal, participativo y operativo. Su periodo de ejecución suele ser de un máximo de 5 años, diferenciando actuaciones a corto plazo ( 1 año), medio (2-3 años) o largo plazo (4-5 años). Su implantación efectiva entraña una serie de problemas, algunos comunes a todos los municipios (estructuras municipales muy departamentalizadas; falta de formación e implicación de los técnicos municipales; escasa funcionalidad del PAL como instrumento efectivo de gestión; insuficiente liderazgo y consenso político; dificultades para mantener un proceso participativo) y otros con mayor incidencia en los de menor tamaño (inexistencia de recursos humanos para el impulso del proceso; escasez de presupuesto; existencia de acciones que superan la capacidad de gestión municipal).

Desde el Departamento de Ordenación del Territorio y Medio Ambiente se ha trabajado también en la elaboración de un listado de indicadores de sostenibilidad comunes para todos los municipios: son 12 , en su mayoría de carácter medioambiental, tal como se especifica en el anexo 1. Este listado puede ser complementado con otros de carácter específico en función de las necesidades de cada municipio. El mismo Departamento ofrece en la Guía metodológica para el cálculo de Indicadores de Sostenibilidad Local en la Comunidad Autónoma del País Vasco. Indicadores de Agenda Local 21 una propuesta de 20 indicadores específicos que incluye tanto indicadores medioambientales como socioeconómicos (IHOBE, 2003).

Una vez aprobado el PAL, el trabajo en grupo puede continuar si los municipios optan por integrarse en Udalsarea 21 (Red Vasca de Municipios hacia la Sostenibilidad). Esta red se constituyó en el año 2002 con el objetivo de coordinar y cooperar en la ejecución de los PALs, contribuyendo de este modo a dinamizar las A21Ls de los municipios adscritos a dicha red, a la que pertenecen 66 (mapa 2). También pertenecen a ella las Diputaciones Forales de las tres provincias, la Asociación de Municipios Vascos (EUDEL) y el Departamento de Ordenación del Territorio y Medio Ambiente /IHOBE. Esta última sociedad asume la Secretaría Técnica de la red y por tanto se encarga de dinamizar y coordinar las tareas que implica el trabajo en grupo así como de realizar todas las labores administrativas derivadas de la gestión de la misma. La incorporación a Udalsarea 21 supone el cumplimiento de los siguientes requisitos: 
a) Un compromiso con la sostenibilidad explicitado en la ratificación del Compromiso por la Sostenibilidad del Pais Vasco y/o la Carta de Aalborg. Hasta el momento 74 municipios se han adherido al primer documento y 91 al segundo. b) Realización de un diagnóstico ambiental y elaboración de un PAL plurianual. c) Formalización de canales para la participación ciudadana.

En 2004 Udalsarea 21 se adherió al ICLEI (International Council for the Local Environmental Initiatives), impulsor de la Conferencia de Aalborg en 1994 y referente internacional de la sostenibilidad local. También en dicho año se firmó un convenio con la Xarxa de Ciutats i Pobles Cap a la Sostenibilitat de Catalunya con el objetivo de impulsar iniciativas comunes entre municipios vascos y catalanes.

\section{La Agenda 21 Local en Vitoria-Gasteiz}

Aunque son mayoría los municipios vascos que han iniciado el proceso de implantación de la A21L, de muy pocos se puede hacer una valoración de sus logros y fracasos, dado los pocos años transcurridos. El municipio que mejor se presta a dicha valoración es el de Vitoria-Gasteiz pues fue pionero en el País Vasco en su implantación. En la temprana implicación de esta ciudad tuvo un papel destacado el alcalde del momento, José Ángel Cuerda, pues no sólo lideró el proyecto sino que también impulsó la creación de estructuras adecuadas a la mejora ambiental del municipio. Especialmente significativa es la creación a finales de los años 1980 del Centro de Estudios Ambientales (CEA) que nació con el objetivo de impulsar iniciativas de formación medioambiental y que con el tiempo ha incorporado nuevos cometidos educativos, formativos y de investigación. En 1995 pasa a constituirse en organismo autónomo dentro del Ayuntamiento. Se estructura en 4 áreas: Área de Formación y Divulgación; Área de Planificación y Proyectos; Área de Sistemas de Información Territorial y Área de Estudios e Investigación. Su papel ha sido muy relevante en el desarrollo del proyecto de Anillo Verde de la ciudad que ha supuesto el acondicionamiento y mejora de una serie de espacios naturales periurbanos y en la propia A21L.

El proceso se inicia en 1995 con la adhesión de Vitoria-Gasteiz a la Carta de Aalborg y su compromiso por trabajar en el diseño e implantación de la A21L. Fue la primera capital española que asumió dicho compromiso. La invitación a participar en la $2^{a}$ Conferencia de Ciudades Sostenibles de Lisboa supuso un aliciente pues obligó a realizar un primer informe que incluía un diagnóstico de la situación ambiental del municipio y una propuesta de objetivos de mejora y líneas de actuación. En la elaboración del informe participaron los técnicos de los distintos departamentos municipales implicados, quienes en una serie de mesas sectoriales revisaron las políticas y 
actuaciones municipales y propusieron 121 acciones de mejora (Ramilo Araujo, 2001). Por tanto, este primer diagnóstico fue realizado exclusivamente por técnicos municipales y no contó con participación ciudadana. El diagnóstico, las propuestas, así como la metodología fueron debatidas en dicha Conferencia. A la vuelta de Lisboa se realizó una revisión del informe al detectarse que proporcionaba una visión demasiado complaciente de la situación ambiental de la ciudad; además se decidió centrarse únicamente en la vertiente ambiental de la sostenibilidad, relegando para una fase posterior la vertiente socioeconómica. La participación ciudadana no se incorpora al proceso de A21L hasta 1998. Ese año el CEA realiza la "Encuesta sobre el medio ambiente en Vitoria-Gasteiz" que aportará la opinión de los ciudadanos sobre los problemas ambientales, la cual se incorporará al diagnóstico técnico junto con el orden de prioridad en la resolución de dichos problemas. La encuesta se realizó a algo más de 1.100 ciudadanos y era bastante exhaustiva ya que incluía un total de 46 cuestiones, unas de carácter general, con las que se pretendía conocer la opinión acerca de la calidad de vida y del medio ambiente en la ciudad y su evolución en los últimos diez años, y otras muchas más de carácter específico sobre los siguientes temas: urbanismo, contaminación urbana, tráfico y transporte, agua, industria, residuos, medio rural y naturaleza, información, educación y participación ciudadana, para acabar examinando el comportamiento de los ciudadanos en relación con los problemas medioambientales. Los resultados manifestaban una clara satisfacción con la evolución de la calidad de vida urbana; se afirmaba que los jardines eran los elementos que más habían mejorado mientras que el tráfico se consideraba que había seguido una evolución desfavorable.

Un año antes, en 1997, el Ayuntamiento organiza unas Jornadas Técnicas, Acciones de las Administraciones Locales Europeas hacia el Desarrollo Sostenible, que sirvieron para impulsar el proceso de A21L no sólo en la ciudad sino también en todo el País Vasco. En este momento muchas autoridades y técnicos municipales de otras poblaciones entran en contacto por primera vez con la A21L, con su marco teórico y su método y conocen experiencias que se estaban desarrollando en ciudades europeas. Estas jornadas sirvieron también para la presentación de un nuevo informe, revisado y mejor elaborado que el que se había llevado con anterioridad a Lisboa. Pero el paso decisivo en la implantación de la A21L de Vitoria-Gasteiz se produce en 1998 cuando el Pleno Municipal aprueba con los votos favorables de todas las formaciones políticas un acuerdo que consta de tres partes. La primera es una declaración de consenso para la promoción del desarrollo sostenible en todo los ámbitos de actuación municipales. La segunda enumera el listado de los indicadores de sostenibilidad y en la tercera se recogen las medidas acordadas para la evaluación, seguimiento y difusión periódica de la Agenda 21. Entre ellas se incluye el impulso al Consejo Sectorial de Medio Ambiente como foro de debate y discusión ciudadana y la publicación de un boletín anual de Agenda 21 que exponga los resultados de la evaluación de los indicadores. 
El primer listado de indicadores, algo más de 170 , fue elaborado por una organización no gubernamental, Bakeaz. Tras un periodo de discusión entre técnicos de los distintos departamentos municipales, que habían participado en la elaboración del diagnóstico, representantes políticos y sociales, se consensuó un nuevo listado más sintético con 21 indicadores que se especifican en el anexo 2. En la decisión final se primó el hecho de que fuesen indicadores medibles y de fácil interpretación para los ciudadanos. Desde su aprobación en 1998 ha regido este listado de indicadores pero al cabo de 6 años se procedió a redefinir el sistema, a crear uno nuevo en el que se han introducido criterios comunes y comparables con los de otros sistemas ampliamente utilizados y aceptados. La incorporación de Vitoria-Gasteiz en el año 2002 a Udalsarea ha supuesto la aceptación de los indicadores comunes a todos los municipios vascos, adaptándolos a las características de la ciudad; también se han tomado como referente los indicadores comunes europeos. El resultado es un nuevo inventario de 35 indicadores que pretenden reflejar las interacciones de los aspectos ambientales, económicos y sociales. De nuevo se llegó a un consenso entre los técnicos municipales y los representantes políticos y este nuevo listado fue aprobado por unanimidad. Al incorporar indicadores de carácter social y económico, la Agenda deja de ser meramente ambientalista. Los 35 indicadores se agrupan en 11 áreas temáticas que se especifican en el anexo 3 . El aumento del número de indicadores implica una evaluación más a fondo de las condiciones ambientales y sociales del municipio, y por tanto un mejor conocimiento de la realidad. Esto permite jerarquizar los problemas y planificar mejor las actuaciones. En definitiva, se están dando nuevos pasos en el compromiso con la sostenibilidad, ya que la. A21L es un proceso dinámico, abierto, que atiende a los problemas según vayan surgiendo. Casi todos los apartados temáticos se han ampliado con nuevos indicadores. Los mayores cambios se advierten en el apartado de tráfico y transporte, donde se han introducido parámetros que permiten conocer la movilidad habitual y los medios de transporte utilizados por los ciudadanos; en el del agua se incorporan otros relacionados con la demanda y con el rendimiento de la red de distribución de agua potable; en urbanismo y territorio, se introduce un indicador sobre el uso sostenible del suelo; en el apartado de salud y riesgos ambientales se han añadido otros dos con el fin de controlar la evolución de los accidentes de tráfico y atropello de peatones; y por supuesto, destaca la presencia de un nuevo apartado, el del medio socioeconómico, que representa el interés explícito por estos temas que apenas se tenían en cuenta en el listado anterior.

El seguimiento de los indicadores compete a distintos departamentos pero el protagonismo principal recae en el Área de Gobierno de Medio Ambiente a la que pertenecen el propio Departamento de Medio Ambiente, el de Salud y Consumo, la empresa municipal AMVISA (Aguas Municipales de Vitoria, S.A.) y el Centro de Estudios Ambientales (CEA). A esta macroárea corresponde la autoría exclusiva del Plan de Acción Ambiental de la Agenda 21 2002-2007. Este Plan se concibe como un instrumento para mejorar la ordenación, coordinación y optimización de los esfuerzos y acciones promovidas por los departamentos y organismos que componen el 
Área de Medio Ambiente. Debe pues entenderse este Plan como un desarrollo más completo y comprometido de la A21L. En su elaboración participaron los técnicos del Área, quienes identificaron las actividades que consideraban que debían desarrollarse entre 2002-2007 para mejorar la situación medioambiental del municipio. También se contó con la participación ciudadana expresada a través del Consejo Sectorial del Medio Ambiente. El Plan se estructura en áreas temáticas, las mismas en las que se agrupan los indicadores ambientales de sostenibilidad. Para cada área temática se establecen unos objetivos, unas acciones a ejecutar y un sistema de evaluación. Sobre algunos temas, el Área de Medio Ambiente dispone de competencias para desarrollar las acciones propuestas: es el caso de contaminación urbana, agua, residuos, naturaleza y biodiversidad e información, educación y participación ciudadana. El resto de las áreas temáticas consideradas, como salud y riesgos ambientales, energía, industria, tráfico y transporte y urbanismo, competen a otras Áreas y Departamentos municipales, pero dada la incidencia que tienen en el medio ambiente son también consideradas en este Plan aunque con un enfoque complementario. En el paso del ecuador de la puesta en marcha de este Plan se está realizando una revisión del mismo pues se ha detectado que algunos de los indicadores de seguimiento resultan difíciles de medir.

Un pilar básico de la A21L es la participación ciudadana como queda expresado en el capítulo 28 del Programa de Agenda 21: "cada autoridad local debería iniciar un diálogo con sus ciudadanos, organizaciones locales y empresas privadas y aprobar una programa 21 local". En el caso de Vitoria, la participación pública en los inicios del proceso quedó reducida, como se ha señalado, a la elaboración de una encuesta. Algunos autores justifican la escasa participación inicial por el desconocimiento de método que posibiliten incorporar la voz de los ciudadanos (Ramilo Araujo, 2001). En 1999 se pone en marcha el Consejo Sectorial del Medio Ambiente, cumpliendo el Acuerdo Plenario Municipal sobre la Agenda 21 del año anterior. El Consejo se constituye como órgano consultivo en el que la representación ciudadana se manifiesta a través de colectivos sociales e instituciones, a la vez que permanece abierto a toda organización que quiera integrarse en él. En 2005 el listado teórico de participantes suma 48 colectivos de todo tipo. En él se incluyen los grupos políticos municipales, asociaciones vecinales, grupos ecologistas y asociaciones proteccionistas, sindicatos, colegios profesionales, centros universitarios, instituciones provinciales y autonómicas, y otras entidades de diverso tipo. La participación numérica en las sesiones de debate normalmente es más reducida. La asistencia técnica la proporciona el Área de Medio Ambiente. El Consejo se ha dotado de un reglamento de funcionamiento interno que se ha renovado en el año 2004. Puede funcionar por grupos de trabajo surgidos del propio Consejo para debatir sobre aspectos temáticos concretos; en el año 2004 sólo funcionó el Grupo del río Zadorra, creado para debatir el proyecto de recuperación de dicho río. El Consejo participó en el Plan de Acción Ambiental y en sus sesiones plenarias se han tratado temas muy diversos y de gran trascendencia para la ciudad. Aunque tiene un carácter consultivo, los políticos responsables de 
medio ambiente incorporan con frecuencia su opinión a las políticas desarrolladas. También está sirviendo para avalar muchos proyectos municipales.

Los boletines anuales de la Agenda 21 publicados por el Ayuntamiento recogen los resultados de la evaluación periódica de los indicadores. Es con esta información con la que se pretende realizar una primera valoración del proceso de A21L y estimar si el municipio avanza hacia un modelo sostenible, apuesta que se inició diez. años atrás. La valoración será aproximada ya que de algunos indicadores no se dispone de información desde 1998. Por otra parte hay que señalar que, si bien la mayoría de indicadores cuenta con mediciones anuales, en otros el seguimiento se realiza cada dos o incluso cada cuatro años. Los indicadores se han clasificado en cuatro categorías: 1) los que evolucionan de forma positiva aunque en algún caso presenten retrocesos puntuales; 2) los que dibujan una tendencia negativa; 3) los que no expresan hasta el momento una tendencia claramente definida ya que experimentan avances y retrocesos o se mantienen estancados; 4) aquellos indicadores de los que se carece de información necesaria para su evaluación.

En la primera categoría se incluyen los siguientes indicadores:

a) Número de viajeros que utilizan el autobús urbano. Excepto un año, todos los demás han experimentado incrementos moderados del número de viajeros. En el período analizado, 1998-2004, la tasa de crecimiento anual del número de viajeros ha sido del $0,48 \%$.

b) Demanda total de agua. En el periodo considerado se ha experimentado un ahorro de 28 litros por habitante y día, siendo la demanda del año más reciente de 289 litros.

c) Rendimiento de la red de distribución de agua potable. El éxito en este indicador es indudable ya que se ha pasado de una eficiencia del 76,9\% al 87,6\%, cifra muy cercana al óptimo.

d) Número de viviendas que han conseguido el certificado de eficiencia energética. El porcentaje de viviendas certificadas asciende al 1,14\% del total, valor que debería aumentar considerablemente en el futuro.

e) Número de empresas con certificado de gestión ambiental. Las empresas que acreditan un certificado, sea el de la norma internacional ISO 14001 o el del sistema europeo EMAS, han pasado de 2 en el año 1998 a 79 en 2004.

f) Generación de residuos domésticos y comerciales. La tendencia apunta una ligera disminución de su peso que se sitúa en $0,56 \mathrm{~kg} / \mathrm{hab} /$ día en el caso de los domésticos y en 0,15 en el de los comerciales en el año 2004 . 
g) Reciclaje de residuos sólidos urbanos. Su evolución positiva ha sido muy clara y constante pues del $9 \%$ de residuos reciclados en 1998 se ha pasado al $24 \%$ seis años más tarde.

h) Número de especies de aves acuáticas que nidifican en el municipio. La evolución, aunque positiva, es modesta.

i) Estado ecológico de los cursos fluviales (índice BMWP').Los tres puntos de control fluvial establecidos, dos en el río Zadorra y uno en el Alegría, señalan datos que, con altibajos interanuales, se pueden calificar de favorables.

j) Número anual de atropellos a peatones en ámbito urbano por cada 10.000 habitantes. En 1999 se registró una tasa de atropellos de 9,51, cifra que se redujo a 5,72 en el año 2004.

k) Número de consultas de información ambiental. Se mide a través del número de accesos a información y documentación ambiental y del número de consultas dirigidas a los servicios técnicos municipales. Los resultados conjuntos confirman un creciente interés ciudadano por el medio ambiente.

1) Participación escolar en las actividades de educación ambiental organizadas por el Ayuntamiento. Este indicador se ha incluido en esta categoría porque, a pesar de que el número absoluto de escolares que participan en programas educativos ha disminuido en los últimos años, su porcentaje se ha incrementado.

m) Gasto per capita del presupuesto municipal destinado a la cooperación internacional para el desarrollo. Los 9,66 euros per cápita que se dedicaban en 2004 a este propósito eran 1,47 euros superior a la cantidad de 1999.

n) Tasa de paro. El periodo analizado coincide con una coyuntura favorable a la creación de empleo que se concreta en un descenso del paro de 7,75 puntos (la tasa de 2004 era de $6,15 \%$ ).

o) Número de días al año en que se registra una calidad del aire regular, mala o muy mala. Su inserción en esta categoría resulta dudosa. En los primeros años aumentó el número de días con mala calidad de aire hasta llegar a 117 en 2001; con posterioridad la situación ha mejorado y dicha cifra se ha rebajado a 74 en 2004 , lo que se achaca a una mayor implicación ambiental por parte de la industria;

Los indicadores que han tenido una evolución desfavorable son los siguientes:

a) Intensidad de tráfico. El número de vehículos que transitan en los días laborables por 6 de las calles más céntricas de la ciudad está aumentando desde 1998. 
b) Consumo doméstico de electricidad y gas natural. La tendencia es la contraria a la deseada puesto que el consumo sigue su curva ascendente. El consumo de gas natural por habitante y año ha pasado de $2.387 \mathrm{kwh}$ en 1999 a 2.979 en $2004 \mathrm{y}$ en el caso de la electricidad de 924 en 1998 a 1.125 en 2004.

c) Tasa de dependencia demográfica. La disminución de la fecundidad y de la mortalidad está propiciando un envejecimiento creciente de la población lo que da lugar a un mayor grado de dependencia demográfica.

Los indicadores que no muestran una tendencia clara en su evolución son los siguientes:

a) Número de veces al año en que se supera el umbral de información a la población para el Ozono. Dado que el número de situaciones de alarma han sido sólo dos en los seis últimos años, no es posible observar ninguna tendencia.

b) Carga orgánica que aporta la ciudad al río Zadorra. Este indicador que analiza la Demanda Química de Oxígeno (DQO), ha experimentado sucesivos altibajos desde 1998.

c) Consumo doméstico del agua. También este indicador se caracteriza por sucesivos incrementos y disminuciones. El año 2004 el consumo era de 130,8 litros/habitante/día.

d) Número anual de accidentes de tráfico en ámbito urbano por cada 1.000 vehículos. De nuevo la curva evolutiva de este indicador proporciona una imagen de dientes de sierra.

e) Porcentaje del presupuesto municipal destinado a la cooperación internacional para el desarrollo. Desde 1999 el porcentaje ha experimentado sucesivos vaivenes aunque se ha mantenido en todo caso por encima del $0,7 \%(0,94 \%$ en el 2004).

f) Porcentaje de familias receptoras del salario social. El indicador expresa el peso de las familias que se benefician del salario social respecto del total de las residentes en el municipio. Su evolución muestra un constante crecimiento que ha pasado de una proporción del 2,53\% en el 2001 al 3,80\% en el 2004. A pesar de una clara evolución al alza, se ha preferido incluirlo en esta categoría por la interpretación ambivalente que puede darse de los datos anteriores. Un aumento de las familias receptoras del salario social puede interpretarse de forma positiva (estaríamos ante una sociedad más solidaria con los más necesitados), pero también de forma negativa (una sociedad desarrollada de la que quedan excluidas cada vez más personas).

g) Distribución sectorial de la demanda total del agua. Este indicador recoge los porcentajes que sobre la demanda total de agua corresponden al consumo domés- 
tico, al consumo de servicios e industria y al consumo municipal. Los datos disponibles desde 1999 muestran una gran estabilidad: un 17-18\% es consumo municipal, alrededor del $30 \%$ es consumo de servicios e industria y en torno al $52 \%$ es consumo doméstico.

El último grupo de indicadores lo integran aquellos de los que sólo se dispone de datos de escasos años, en ocasiones de uno solo. Son los siguientes:

a) Población residente en calles expuestas durante las 24 horas del día a niveles de ruido exterior superiores a los deseables como objetivo de calidad.

b) Población residente en calles expuestas durante el periodo nocturno a niveles de ruido exterior superiores a los deseables como objetivo de calidad. La única fuente disponible para medir la contaminación sonora, diurna y nocturna, es el mapa de ruidos que se realizó en el año 2003, por tanto no es posible valorar su evolución.

c) Movilidad local y transporte de pasajeros. No se dispone más que de los datos proporcionados por una encuesta realizada en el año 2001.

d) Superficie dedicada a infraestructuras de transporte. Los únicos datos que se han facilitado corresponden al Mapa de Usos del Suelo del año 2003. Aunque tampoco puede observarse una evolución, un dato significativo es que en el mapa de dicho año se señalaba que el $71,2 \%$ correspondía a superficie de uso de vehículos privados frente al $3 \%$ para uso del transporte público colectivo. El resto de la superficie se dedicaba a uso peatonal $(25 \%)$ y de bicicarriles $(0,8 \%)$.

e) Depuración de aguas residuales. Este indicador analiza el porcentaje de viviendas del municipio conectadas a sistemas de tratamiento de aguas residuales. Sólo existe información de los años 2003 y 2004 , insuficiente para definir tendencias. Con todo, los datos muestran una situación difícilmente mejorable ya que sólo el $0,07 \%$ de las viviendas carecen de sistema de depuración.

f) Acceso a servicios básicos y zonas públicas abiertas. La información disponible es insuficiente para definir tendencias aunque los datos muestran en general bastante estabilidad. La accesibilidad ha mejorado algo respecto a los servicios sanitarios y farmacias y ha experimentado un pequeño deterioro en el acceso a otros servicios como los educativos y culturales.

g) Uso sostenible del suelo. Es uno de los nuevos indicadores que en el futuro va a ser de los que mayor interés adquieran cara a la sostenibilidad urbana y territorial. Su resolución es compleja ya que consta de seis subindicadores, pero la información disponible en la actualidad es todavía limitada. 
h) Superficie agrícola municipal que desarrolla agricultura ecológica. Con este enunciado este indicador se incorpora en el año 2004 y por lo tanto nada se puede señalar sobre su evolución. Anteriormente se medía la superficie de terrenos agrícolas que se acogían a programas de prácticas agroambientales. Los datos reflejan una escasa aceptación ya que apenas 26 has se acogian a este programa ambiental sin que dicha superficie mostrase modificaciones.

i) Porcentaje de fumadores. No existen datos suficientes para determinar su evolución.

j) Satisfacción ciudadana con la comunidad local. Hasta el momento sólo se dispone de la información aportada por una encuesta realizada en el año 2001 que mostraba una ciudadanía bastante satisfecha con su ciudad salvo en el tema de la vivienda.

\section{Conclusiones}

Por lo que respecta al conjunto del País Vasco las actuaciones llevadas a cabo por el Departamento de Ordenación del Territorio y Medio Ambiente del Gobierno Vasco merecen una valoración positiva, ya que han mostrado un indudable compromiso político con el proceso de A21L y un fomento del trabajo agrupado en redes (Udaltalde, Udalsarea). Este esfuerzo se ha reflejado en el elevado número de municipios implicados en el desarrollo de la Agenda 21. No obstante, cabe mencionar ciertos riesgos compartidos por los procesos excesivamente tutelados y desarrollados de arriba abajo. Uno de ellos consiste en una cierta homogeneidad en los diagnósticos y programas que menoscabe las particularidades locales. Así mismo el seguimiento de los protocolos emanados de instancias superiores puede llegar a minusvalorar aportaciones directas de la base ciudadana. Cara al futuro es de temer que, al menos en algunos lugares, el previsible debilitamiento de la ayuda tutelada conduzca a un desinterés por la buena marcha de la situación ambiental.

El análisis más detallado del caso vitoriano refleja con claridad la trascendencia que adquiere la voluntad política para el desarrollo de la A21L. Dicha voluntad se ha manifestado en un liderazgo social y en la puesta a punto de mecanismos diversos de organización y gestión. Entre ellos destaca el protagonismo otorgado a la macroárea de Medio Ambiente, poco frecuente en otros ayuntamientos (Aguado \& Echebarria, 2003), dentro de la cual sobresale la fortaleza y eficacia mostradas por el Centro de Estudios Ambientales. La preeminente situación de este Área dentro del Ayuntamiento le capacita para ejercer una influencia notable en otros departamentos municipales, facilitando la necesaria transversalidad administrativa que precisan los 
temas ambientales y de sostenibilidad, transversalidad que siempre es mejorable. Fruto de la colaboración interdepartamental es el Sistema de Información Ambiental que posee una gran riqueza informativa, puesta al día y accesible a la ciudadanía a través de la página web del Ayuntamiento. A pesar de los indudables esfuerzos realizados en el estímulo a la participación ciudadana, ésta debería superar su estructura sectorial centrada en el medio ambiente, sesgo que ha caracterizado a todo el proceso, para abarcar el conjunto de temas que compete a la sostenibilidad. Por otro lado, se precisarían ideas y procedimientos innovadores que mejoren dicha participación y amplíen su base social (Castiella \& Satorras, 2002).

La evolución hacia la sostenibilidad mostrada por los indicadores de la A21L presenta claroscuros, aunque valorada en su conjunto se puede calificar de positiva ya que la situación de aquellos temas que no han evolucionado de forma adecuada podría haber sido peor si no hubiese habido actuaciones que contrarrestasen su inercia. En concreto una serie de indicadores presentan una tendencia favorable entre los que cabe destacar el ciclo urbano del agua y el reciclaje de residuos. Hay otros que han seguido una tendencia negativa, dos de ellos de gran transcendencia dentro de los parámetros de sostenibilidad: movilidad urbana y transporte y consumo de energía. En este punto resalta un hecho de hondo calado. Gran parte de los indicadores que evolucionan de forma satisfactoria obedecen a actuaciones que dependen directamente de la administración pública sin que el ciudadano tenga una implicación directa en su manejo, aunque se beneficie de su mejora. Por el contrario algunos de los indicadores desfavorables mantienen una relación directa con el comportamiento social, como es el caso de los ya citados de la energía y la movilidad. Este hecho permite concluir que el camino hacia estados más sostenibles depende en gran medida de la mentalidad y la escala de valores de los ciudadanos, que se manifiestan en su comportamiento diario, reflejo del modelo socioeconómico de consumo dominante. Sin una modificación de las escalas de valor vigentes no son previsibles mejoras cualitativas profundas. Aunque la voluntad política local mantenga su compromiso con la sostenibilidad, como demuestra su adhesión, junto con otras 49 ciudades de todo el mundo, a los Acuerdos Urbanos del Medio Ambiente-Declaración de Ciudades Verdes adoptados el 5 de junio de 2005 en San Francisco, si la presión popular no se dirige hacia el mismo propósito, la actuación política se verá entorpecida hasta el punto de volverse inoperante o, en el escenario más probable, acordará medidas no sostenibles pero del agrado de una mayoría social.

\section{Bibliografía}

Aguado, I \& Echebarria, C. (2003): "Medio Ambiente y desarrollo sostenible en España", Boletín Económico de ICE, $\mathrm{n}^{\circ}$ 2.786, pp. 21-30.
Área de Medio Ambiente (1999-2005): Agenda 21, Boletín. Vitoria-Gasteiz bacia un desarrollo sostenible, Ayuntamiento de Vitoria-Gasteiz. 
Brunet P.J.; Almeida, F.; Coll, M. (2005): "Agenda 21: Subsidiariedad y Cooperación a favor del Desarrollo Territorial Sostenible", Boletín de la Asociación de Geógrafos Españoles, no 39 , pp. 423-446.

Castiella, T. \& Satorras, M. (2002): "La participación en el marco de la innovación local. El caso de la Agenda 21 de Barcelona", Análisis Local, $\mathrm{n}^{\circ} 44, \mathrm{~V}$, pp. 35-41.

Departamento de Ordenación del Territorio y Medio Ambiente, www.ingurumena.net.

Echebarría, C.\& Aguado, I. (2002): "La gestión territorial local desde la perspectiva medioambiental. La ciudad de VitoriaGasteiz", Ciudad y Territorio. Estudios Territoriales, XXXIV (132), pp. 325-342.

Echebarría, C.\& Aguado, I. (2004): "Agenda 21 Local: estudio bibliográfico y análisis de una experiencia", Ciudad y Territorio. Estudios Territoriales, XXXVI (139), pp. 67-88.

Espinoza Guerra, L. E. (2001): "Ciudad y medio ambiente: la Agenda 21 Local", Sistema 162-163, pp. 219-231.
IHOBE (2002): Estrategia ambiental vasca de desarrollo sostenible 2002-2020, Bilbao, Gobierno Vasco. Departamento de Ordenación del Territorio y Medio Ambiente.

IHOBE (2003): Guía metodológica para el Cálculo de Indicadores de Sostenibilidad Local en la Comunidad Autónoma del Pais Vasco, Bilbao, Gobierno Vasco. Departamento de Ordenación del Territorio y Medio Ambiente.

IHOBE (2004): Agenda Local 21. Guía para la puesta en marcha de Planes de Acción Local, Bilbao, Gobierno Vasco. Departamento de Ordenación del Territorio $y$ Medio Ambiente.

Ramilo Araujo, M. C. (2001): "De la sostenibilidad ambiental a la transversalidad de las políticas públicas. La Agenda Local 21 en Vitoria-Gasteiz: Logros y retos", Inguruak, 21, pp. 121-137.

Riego Artigas, P. (2004): La Agenda 21 Local, Madrid, Mundi-Prensa.

\section{Anexo 1. Indicadores de sostenibilidad comunes de los municipios vascos}

Medio Ambiente: Territorio y Planeamiento Indicador 1: Disponibilidad de zonas públicas abiertas y de servicios en el municipio.

Indicador 2: Uso sostenible del suelo.

Medio Ambiente: Movilidad y Transporte

Indicador 3: Movilidad local y transporte de pasajeros.

Indicador 4: Distribución de la superficie municipal dedicada a infraestructuras de transporte.

Medio Ambiente: Recursos Naturales

Indicador 5: Consumo de agua.

Indicador 6: Consumo de energía.

\section{Medio Ambiente: Residuos}

Indicador 7: Generación y gestión de residuos. Indicador 8: Vertidos al agua.

Medio Ambiente: Integración del Medio Ambiente en las actividades municipales Indicador 9: Sistemas de gestión medioambiental en el municipio.

\section{Medio Social y Económico}

Indicador 10: Pobreza y exclusión social. Indicador 11: Tasa de paro.

Indicador 12: Satisfacción de la ciudadanía con la comunidad local. 


\section{Anexo 2. Sistema de indicadores de Vitoria-Gasteiz aprobado en el acuerdo municipal} de 1998

\section{Contaminación urbana}

1. Número de días al año en que se registran calidades de aire "regular" y/o "mala".

1. Número de veces al año que se supera el umbral de información a la población para el Ozono.

2. Población expuesta durante las horas diurnas y nocturnas a niveles de ruidos superiores a los recomendados por la Organización Mundial de la Salud.

\section{Tráfico y transporte}

3. Número de viajeros que utilizan el autobús urbano.

4. Número de vehículos que circulan diariamente por las principales calles de acceso al centro de la ciudad.

\section{Agua}

5. Consumo doméstico de agua por habitante y día.

6. Porcentaje de agua que se recicla,

7. Proporción de carga orgánica aportada al río Zadorra por la ciudad.

Energía

8. Consumo doméstico de gas y electricidad por habitante y año.

\section{Industria}

9. Número de empresas de la ciudad que han conseguido el certificado de gestión ambiental.

\section{Residuos}

10. Peso de residuos domésticos producidos por habitante y día.

11. Porcentaje de residuos reciclados frente a los producidos.

\section{Urbanismo}

12. Número de licencias concedidas para la rehabilitación de viviendas.

13. Porcentaje de población que tiene acceso a pie $(<1 \mathrm{~km})$ a los servicios básicos.

14. Número de edificios que han conseguido el certificado de eficiencia energética.

\section{Naturaleza y biodiversidad}

15. Número de especies de aves acuáticas reproductoras existentes en el Municipio.

16. Superficie $\left(\mathrm{m}^{2}\right)$ de áreas degradadas recuperadas.

17. Superficie de terrenos agrícolas que se acogen a algún programa de prácticas agrícolas ambientales.

Salud y riesgos ambientales

18. Número de fumadores.

Información, educación y participación ciudadana

19. Número de consultas de información ambiental.

20. Porcentaje de la población escolar que accede a programas de educación ambiental organizados por el Ayuntamiento.

\section{Anexo 3. Sistema de indicadores vigente en el municipio de Vitoria-Gasteiz}

\section{Contaminación urbana}

1. Número de días al año en que se registra una calidad del aire "regular, "mala" o "muy mala".

2. Número de veces al año en que se supera el umbral de información a la población para el Ozono.

3. Población residente en calles expuestas durante las 24 horas del día a niveles de ruido exterior superiores a los deseables como objetivo de calidad
4. Población residente en calles expuestas clurante el período nocturno a niveles de ruido exterior superiores a los deseables como objetivo de calidad.

\section{Tráfico y transporte}

5. Movilidad local y transporte de pasajeros.

5a. Número medio de desplazamientos que cada habitante realiza a cliario.

5b. Distancia media diaria recorrida por cada habitante. 
5c. Duración media de los desplazamientos diarios de cada habitante.

5d. Porcentaje de viajes sistemáticos y no sistemáticos

5e. Porcentaje de utilización de los diferentes modos de transporte.

6. Número de viajeros que utilizan el autobús urbano.

7. Intensidad del tráfico.

8. Superficie dedicada a infraestructuras de transporte.

Agua

9. Carga orgánica que aporta la ciudad al río Zaclorra.

10. Depuración de aguas residuales.

11. Consumo doméstico de agua.

12. Demanda total de agua.

13. Distribución sectorial de la clemanda total de agua.

14. Rendimiento de la red de distribución de agua potable.

\section{Energía}

15. Consumo doméstico de electricidad y gas natural.

16. Número de viviendas que han conseguido el certificado de eficiencia energética.

\section{Industria}

17. Empresas con certificado de gestión anbiental.

\section{Residuos}

18. Generación de residuos domésticos y comerciales.

19. Reciclaje de residuos sólidos urbanos.

\section{Urbanismo y Territorio}

20. Accesibilidad a servicios básicos y a zonas públicas abiertas.

21. Uso sostenible del suelo.

21a. Suelo artificializado y urbanizable.

21b. Suelos abandonados y potencialmente contaminados.

21c. Intensidad de uso del suelo.

21d. Localización de los nuevos desarrollos. 21e. Restauración de superficies urbanas.

21f. Protección y recuperación del espacio rural y natural.

\section{Naturaleza y biodiversidad}

22. Número de especies de aves acuáticas que nidifican en el municipio de VitoriaGasteiz.

23. Superficie agrícola municipal que desarrolla agricultura ecológica.

24. Estado ecológico de los cursos fluviales (indice BMWP').

Salud y riesgos ambientales

25. Porcentaje de fumadores (habituales u ocasionales)

26. Número anual de accidentes de tráfico en ámbito urbano por cada 1.000 vehículos.

27. Número anual de atropellos a peatones en ámbito urbano por cada 10.000 habitantes.

Información, educación y participación ciudadana

28. Número de consultas de información ambiental.

28a. Número de accesos a información y documentación ambiental.

28b. Número de consultas dirigidas a los Servicios Técnicos Municipales.

29. Participación escolar en las actividades de educación ambiental organizadas por el Ayuntamiento de Vitoria-Gasteiz.

\section{Medio socioeconómico}

30. Satisfacción ciudadana con la comunidad local.

31. Porcentaje del presupuesto municipal clestinado a la cooperación internacional para el desarrollo.

32. Gasto per capita del presupuesto municipal destinado a la cooperación internacional para el desarrollo.

33. Tasa de dependencia demográfica.

34. Porcentaje de familias receptoras del salario social.

35. Tasa de paro. 

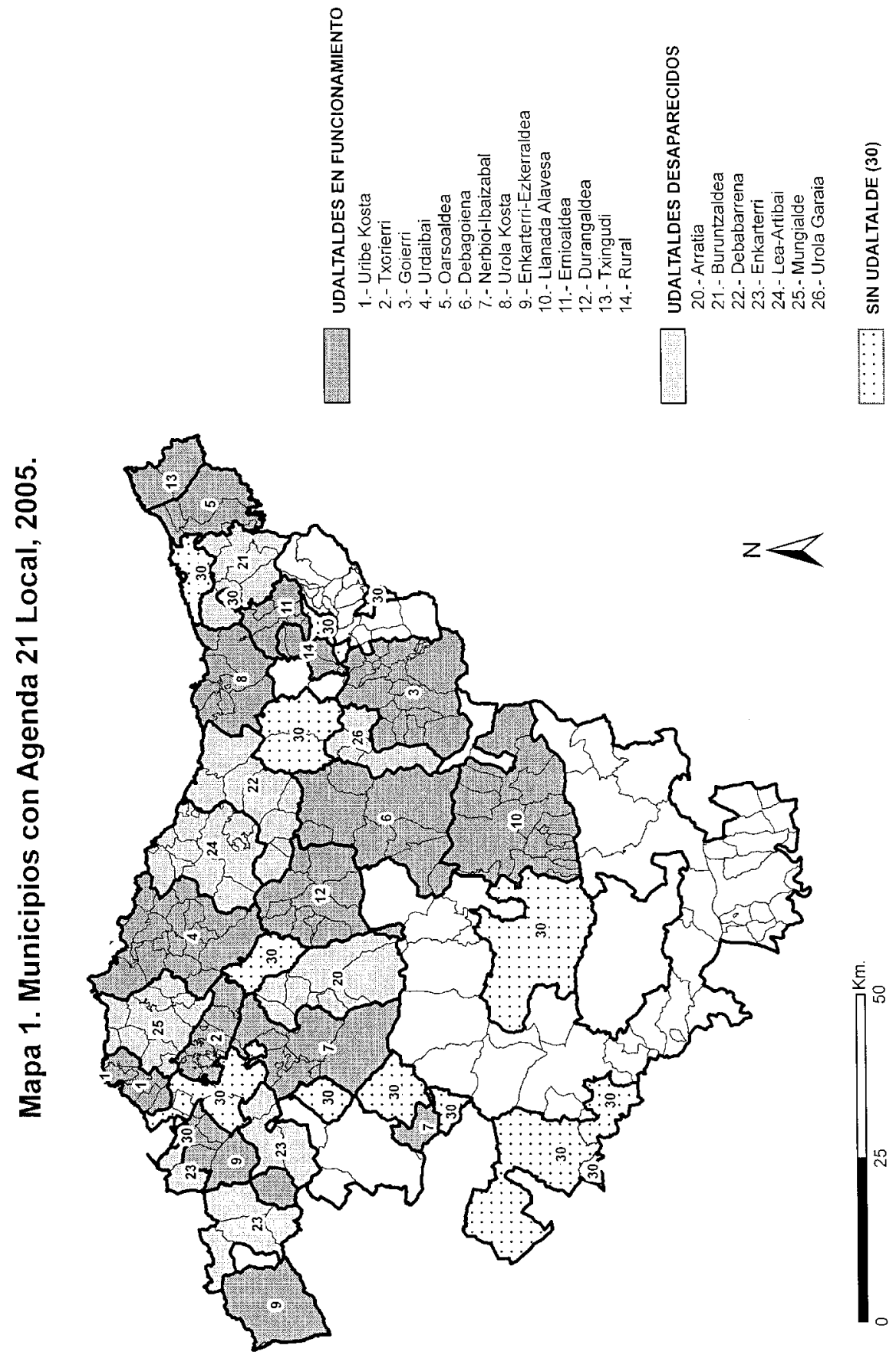


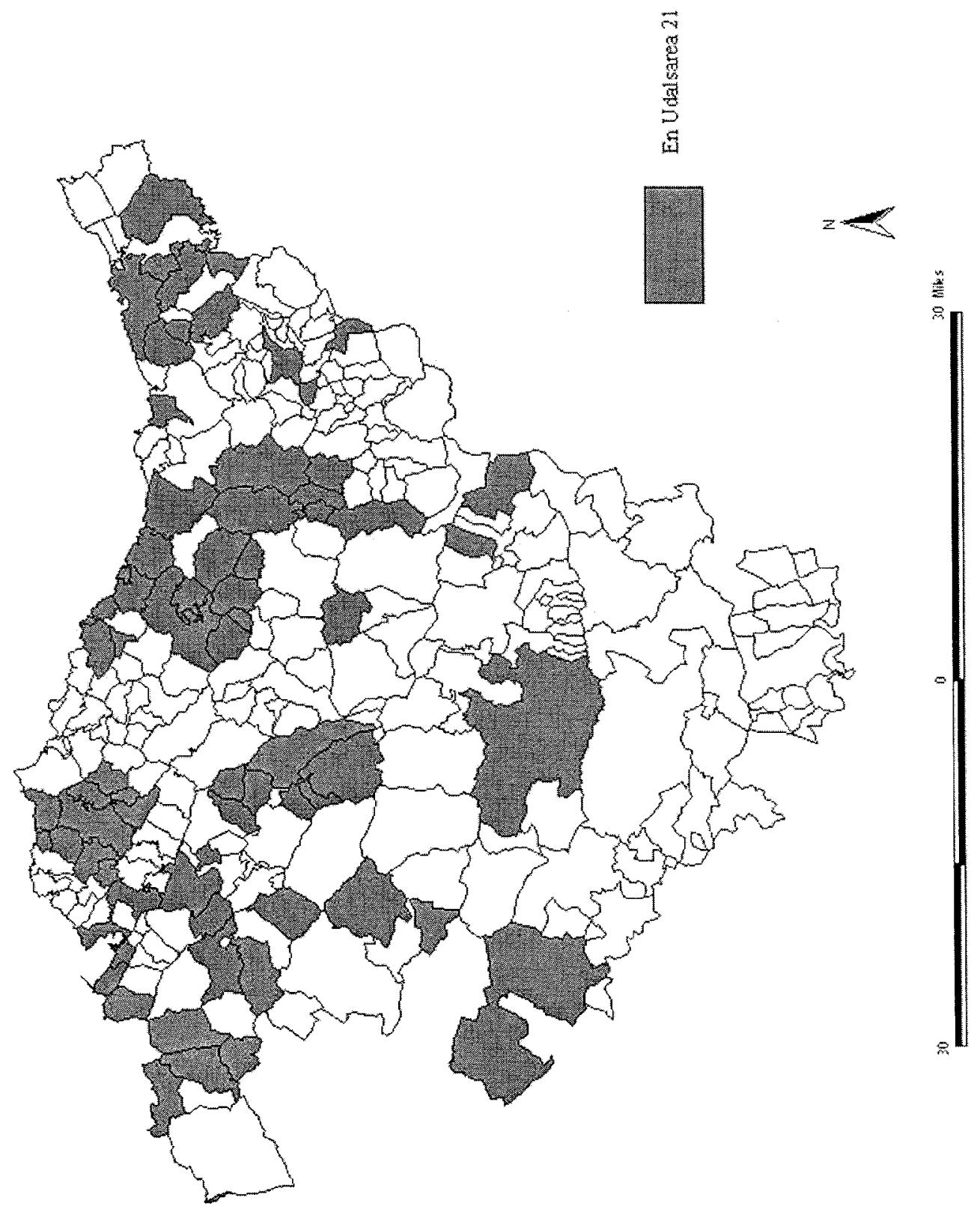

